\title{
The Relationship between High Sensitive C-reaction Protein (hs-CRP) and Diastolic Heart Function in Diabetes Mellitus Type II
}

\author{
Samiramis Ghavam ', Mohammad Reza Hafezi Ahmadi ${ }^{2,5}$, Aslan Mosavi ${ }^{3}$, Elham Elhamdoost ${ }^{3}$ \\ and Behrang Kazeminezhad ${ }^{4^{*}}$ \\ 'Department of Cardiology, Ilam University of Medical Sciences, Ilam, Iran \\ 2Department of Pathology, Ilam University of Medical Sciences, Ilam, Iran \\ 3llam University of Medical Sciences, Ilam, Iran \\ ${ }^{4}$ Department of Pathology, Clinical Research Development Center, Shahid Modarres Hospital, \\ Shahid Beheshti University of Medical Sciences, Tehran, Iran ;dkazeminezhad@gmail.com \\ ${ }^{5}$ Biotechnology and Medical Plants Research Center, Ilam University of Medical Sciences, Ilam, Iran
}

\begin{abstract}
Among several inflammatory markers, high sensitive C-reaction protein (hs-CRP) is outstandingly observed in diabetic individuals. Serum hs-CRP is the main marker of inflammation whose levels independently predict the risk of cardiovascular events, and it has a prognostic value in heart patients. On the other hand, diabetes can lead to diastolic dysfunction of the heart. Diastolic dysfunction can cause symptoms of exertional dyspnea, which restricts the patient's activity. It is likely to predict diastolic dysfunction by screening through hs-CRP. The present investigation was a case-control study that was carried out on 52 patient diagnosed with diabetes mellitus type II. After the demographic data were recorded, and following the collection of data on the patients' history, physical examination, and para-clinical measures, individuals who had factors interfering with level of serum hs-CRP (kidney and liver diseases, inflammatory and infectious diseases, peripheral vascular disease, cerebrovascular disease, connective tissue disease, malignant tumor, trauma, consumption of statins, aspirin, ACEI, and fibrates) and diastolic dysfunction (ischemic heart disease, cardiomyopathies, pericardial disease, arrhythmias and valvular disease) were crossed out of the study. Serum hs-CRP was measured by nephelometry method. According to the results of tissue Doppler echocardiography, these patients are divided into two groups: one with diastolic dysfunction and the other without diastolic dysfunction. The serum hs-CRP levels of these patients were compared with each other. Among the participants, $30.8 \%$ were men and $69.2 \%$ were women, 36 individuals (69.2\%) had diastolic dysfunction while 16 (30.8\%) did not. There was a high level of correlation between the level of serumhs-CRP and diastolic dysfunction $(\mathrm{p}=0.02, \mathrm{t}=2.36)$. The results of the present study indicated that there is a correlation between level of serum hs-CRP and diastolic dysfunction, such that the more the level of hs-CRP, the higher probability of diastolic dysfunction existence will be.
\end{abstract}

Keywords: Diastolic Dysfunction, Diabetes Mellitus, hs-CRP

\section{Introduction}

Diabetes mellitus is a metabolic disease whose prevalence has been increased over that last several decades, such that the number of the patients increased from 30 million people in 1985 to 177 million in 2000, and it is predicted this number will reach over 320 million by 2030. Diabetes is one of the major causes of death and is responsible for the death of 3 million people all over the world in $2002^{1}$.

The number of cardiovascular diseases is increasing among diabetic patients. American Heart Association has considered diabetes as a risk factor for cardiovascular diseases and ranked it at the same level as smoking, hypertension, and hyperlipidemia. There is little evidence indicating that better control of blood sugar leads to a decrease in cardiovascular complications in diabetes,

*Author for correspondence 
and better control of blood sugar has not convincingly reduced cardiac mortality. The rate of heart failure is high among diabetic patients, which can be attributed to different reasons including atherosclerosis, hypertension, and heart cell dysfunction due to a chronic increase in hyperglycemia ${ }^{1}$.

Among several inflammatory markers, hs-CRP can outstandingly be seen in diabetic patients. Serum hs-CRP is the major marker of inflammation which is created by the kidney. This protein increases in response to inflammation, injury, and infection ${ }^{2,3}$. Nowadays, the results of a set of prospective epidemiological studies have indicated that levels of CRP can alone predict the risk of cardiovascular events and has a prognostic value in cardiac patients ${ }^{4,5}$. Myocardial fibrosis is the major cause of myocardial stiffness which leads to advanced heart diastolic dysfunction which has a higher prevalence in inflammatory conditions and causes the symptoms of exertional dyspnea and restricts the patient's activity ${ }^{6}$.

In some studies, it is reported that mortality rate in asymptomatic patients who are in diastolic dysfunction phase I during 3-5 years is 5 times more than those with normal diastolic function ${ }^{7}$. Therefore, due to the high prevalence of diabetes and its serious cardiac complication, it is necessary to diagnose diastolic dysfunction as soon as possible so that necessary grounds for reducing the costs and increasing the quality of the patient's life can be established by examining the influential factors.

\section{Materials and Methods}

Fifty-two 20-55-year-old outpatients with diabetes mellitus type II who had referred to Ilam'sHeart Clinic in 2012-13 and had study inclusion criteria were selected. The patients were provided with necessary explanations about the study and their written consent was obtained. Patients who had intervening factors in the level of hs-CRP like kidney and liver diseases, inflammatory and infectious diseases, peripheral vascular disease, cerebrovascular disease, connective tissue disease, malignant tumor, recent injuries, and consumption of ACEI, ASA, statins, and fibrates were crossed from the study. An increase of over $10 \mathrm{mg} / \mathrm{L}$ in hs-CRP is in favor of the existence of an acute inflammation; therefore, patients with a serum hs-CRP level of over $10 \mathrm{mg} / \mathrm{L}$ were crossed out of the study. Those patients who in their para-clinical examinations were known to have factors affecting diastolic function (such as valvular disease, pericardial disease, cardiomyopathies, and ischemic heart disease) were also crossed out of the study.

The questionnaire was aimed at collecting data on age, gender, BMI, disease control and treatment method, disease duration, alcohol consumption, smoking, and family and medical background. ECG was run in order to examine ischemic and rhythm disorders. Afterwards, ischemic heart diseases were checked using exercise test and in some patients using R/O scan. Heart function was examined and recorded using tissue Doppler echocardiography by an echocardiography subspecialist. Patients who had diastolic dysfunction were classified into mild, moderate, and acute groups ${ }^{8}$ (Table 1 ).

Table 1. Classification of diastolic dysfunction

\begin{tabular}{lcccc}
\hline Parameter & Normal & Mild & Moderate & Acute \\
\hline E/A ratio & $1-2$ & $<0.8$ & $0.8-2$ & $\geq 2$ \\
$D T(m s($ & $150-200$ & $>200$ & $150-200$ & $<140$ \\
$E$ Velocity & $10 \leq$ & $<8$ & $<8$ & $<5$ \\
E/E ratio & $8 \geq$ & $<8$ & $9-14$ & $\geq 15$ \\
$I V R T(m / s)$ & $50-100$ & $\geq 100$ & $60-100$ & $\leq 60$ \\
$P V_{S} / P V_{D}$ & $\approx 1$ & $\mathrm{~S}>\mathrm{D}$ & $\mathrm{S}<\mathrm{D}$ & $\mathrm{S}<<\mathrm{D}$ \\
$P V_{a}(m / s)$ & $<0.35$ & $<0.35$ & $\geq 0.35$ & $\geq 0.35$ \\
$a_{\text {dur }}-A_{\text {dur }}(m s)$ & $<20$ & $<20$ & $\geq 30$ & $\geq 30$ \\
\hline
\end{tabular}

In addition to echocardiography (echo), the patients were referred to the laboratory in order to measure the chemical biomarkers and levels of hs-CRP. The level of serum hs-CRP was measured by nephelometry method.

Afterwards, according to the results of the echo, these patients were divided into two groups; one with diastolic dysfunction and one without it. The level of hs-CRP of these patients was compared, and the basic level of serum hs-CRP was classified into four groups: below $0.5 \mathrm{mg} / \mathrm{L}$ (very low-risk group), 0.5-1 mg/L (low-risk group), 1-3 $\mathrm{mg} / \mathrm{L}$ (average risk group), and over $3 \mathrm{mg} / \mathrm{L}$ (severe risk group), and its relationship with different variables was investigated.

\section{Statistics}

The collected data were analyzed using SPSS. t-test and one-way ANOVA were employed to examine the relationship between quantitative variables and the classified ones. Chi-square test was run to check the relationship between the qualitative variables. The relationship between hs-CRP and quantitative variables 
was examined using Pearson's correlation. To check the capacity of hs-CRP in predicting diastolic dysfunction, a logistic regression model was used. The obtained values were reported as Mean $\pm \mathrm{SD}$ and the odd ratio was reported with a confidence distance of $95 \%$. A p-value of below 0.05 was considered to be significant.

\section{Results}

Among the participants, $30.8 \%$ were men and $69.2 \%$ were women. The mean age of the participants was $43.37 \pm 6.49$ years, and only $17.3 \%$ of the patients were below 40 years old.

The results of examining the risk factors for cardiac disease indicated that $23 \%$ of the patients had the family history of premature heart disease among their firstdegree relatives. It was also concluded that $96.2 \%$ of the patients were non-smokers, $100 \%$ of the participants had not consumed alcohol, $25 \%$ had exercised on a daily basis, and $19.2 \%$ had an intervening factor at hs-CRP level (hypertension). The patients' mean BMI was 27.46 \pm 3.81 ; the women had a higher BMI (with an average of $27.55 \pm 4.42$ ) compared to the men. Moreover, most of the patients had a normal diastolic and systolic blood pressure, and the patients' cardiac output was normal (with a mean of 53.17).

According to the results of the tissue Doppler echocardiography, out of the participating patients, 36 people (69.2\%) had and 16 (30.8\%) did not have diastolic dysfunction. In the present study, first, individuals with intervening factors at the level of hs-CRP (including hypertension) were compared in terms of the mean hsCRP. The results of the independent $t$-test indicated that there was no significant difference between the two groups regarding the mean hs-CRP $(\mathrm{p}=0.471)$.

According to the drawn tables, the mean hs-CRP in the group with diastolic dysfunction was $1.46 \pm 0.78$ while it was $0.86 \pm 1.01$ in the group without diastolic dysfunction. The results of the independent $t$-test showed that there was a statistically significant difference between the two groups in terms of mean hs-CRP $(\mathrm{p}=0.02, \mathrm{t}=2.36)$. The mean level of hs-CRP increases with the progress of the phase of diastolic dysfunction and this relationship is statistically significant $(\mathrm{p}=0.021)$.

Pearson correlation coefficient indicated that there was a positive significant relationship between the level of serum hs-CRP and disease duration ( $\mathrm{p}=0.03, \mathrm{r}=0.4)$.
Pearson correlation coefficient also showed that the level of hs-CRP rose with an increase in systolic blood pressure in diabetic patients. This coefficient also indicated that there was no significant relationship between hs-CRP and the variables of BMI, weight, age, $\mathrm{HR}, \mathrm{EF}$, and $\mathrm{DBP}$ ( $p>0.05)$. The results of the independent $t$-test showed that there was no significant relationship between the level of hs-CRP and gender ( $\mathrm{p}=0.139)$.

The results of the Chi-square test showed that there was no significant relationship between diastolic dysfunction and gender $(\mathrm{p}=0.622)$. The independent t-test showed that there was a significant relationship between diastolic dysfunction and age $(\mathrm{p}=0.018)$. Moreover, the results of the independent $t$-test indicated that there was a significant relationship between diastolic dysfunction and disease duration $(\mathrm{p}=0.041)$. They also proved that there was no significant relationship between diastolic dysfunction and the variables of SBP, DBP, BMI, and weight ( $\mathrm{p}>0.05)$.

Table 2. The demographic characteristics of the participants and their correlation with hs-CRP

\begin{tabular}{lcccc}
\hline Variable & Mean & SD & $\begin{array}{c}\text { Pearson } \\
\text { Correlation }\end{array}$ & p-Value \\
\hline Age & 47.37 & 6.493 & 0.063 & 0.656 \\
Disease duration & 5.26 & 3.94 & 0.406 & 0.03 \\
Weight & 74.23 & 1.28 & -0.073 & 0.605 \\
SBP & 118.23 & 9.51 & 0.287 & 0.039 \\
DBP & 74.50 & 9.64 & 0.036 & 0.798 \\
BMI & 27.46 & 3.81 & 0.046 & 0.747 \\
\hline
\end{tabular}

Logistic regression model was employed to examine the relationship between the increase probability of diastolic dysfunction risk and an increase in the level of hs-CRP. This model indicated the relationship between these two variables as:

$\mathrm{e}^{-1 / 05}=\operatorname{Exp}$

This model indicates that with every unit increase in hs-CRP, there will be an increase of 2.85 times in the probability of diastolic dysfunction (Odd ratio $\left(\frac{1}{E x p}\right)=$ $2 / 85)$. Since an increase in the level of hs-CRP leads to an increase in the risk of cardiovascular diseases, the level of serum hs-CRP was divided into four groups: the groups with the level of serum below $0.5 \mathrm{mg} / \mathrm{L}$ was considered as the group with very low risk of cardiovascular diseases, 0.5-1 mg/L as low risk, $1-3 \mathrm{mg} / \mathrm{L}$ as average risk, and over 
$3 \mathrm{mg} / \mathrm{L}$ as severe risk. Since a very limited number of the patients had hs-CRP level of over $3 \mathrm{mg} / \mathrm{L}$, they were also considered in the group with levels of 1-3 mg/L. In order to examine the relationship between the level of hs$\mathrm{CRP}$ as an ordinal variable and diastolic dysfunction, an ordinal logistic regression model was employed.

This model indicated that by increasing the level of hsCRP from one level to another, the probability of diastolic dysfunction increases, such that if the level of the patient's hs-CRP is at the level with average risk, the probability of diastolic dysfunction will be 20.8 times more than that of someone whose serum hs-CRP is normal.

Table 3. The mean of the variables among the participants according to diastolic dysfunction

\begin{tabular}{|c|c|c|c|c|}
\hline D.D. & & Has & $\begin{array}{c}\text { Does not } \\
\text { have }\end{array}$ & $\begin{array}{c}\mathrm{p} \text {-Val- } \\
\text { ue }\end{array}$ \\
\hline Variable & & & & \\
\hline Age (year) & & $49 \pm 6$ & $44 \pm 7$ & 0.018 \\
\hline $\begin{array}{l}\text { Duration of } \\
\text { disease(year) }\end{array}$ & & $6.04 \pm 4.1$ & $3.66 \pm 3.14$ & 0.041 \\
\hline Weight (kg) & רyerng & $2.23 \pm 75 / 02$ & $2.98 \pm 72.58$ & 0.527 \\
\hline hs-CRP (mg/L) & average & $1.47 \pm 0 / 79$ & $0.86 \pm 1 / 02$ & 0.022 \\
\hline DBP (mmHg) & & $74 \pm 8$ & $75 \pm 13$ & 0.751 \\
\hline SBP $(\mathrm{mmHg})$ & & $121 \pm 9$ & $113 \pm 8$ & 0.008 \\
\hline $\mathrm{EF}$ & & $0.42 \pm 55.42$ & $0.58 \pm 55.58$ & 0.83 \\
\hline HR & & $0.83 \pm 70 / 11$ & $4.05 \pm 66.29$ & 0.215 \\
\hline BMI $\left(\mathrm{kg} / \mathrm{m}^{2}\right)$ & & $27.46 \pm 3 / 59$ & $27.47 \pm 4.37$ & 0.995 \\
\hline
\end{tabular}

Table 4. Evaluating the grouping parameter of hs-CRP in the ordinal logistic regression model

\begin{tabular}{lccccc}
\hline hs-CRP & Estimation ability & S.E & Wald & df & OR \\
\hline$<0 / 5$ & & & $14 / 132$ & 2 & \\
$0 / 5-1$ & $-1 / 56$ & $1 / 017$ & $2 / 38$ & 1 & $\frac{1}{0 / 208}=(4 / 8)$ \\
& & & & & \\
$1-3$ & $-3 / 035$ & $0 / 809$ & $14 / 060$ & 1 & $\frac{1}{0 / 048}=(20 / 8)$ \\
\hline
\end{tabular}

Pearson correlation coefficient in the present study indicated that there was a positive significant relationship between systolic blood pressure and hs-CRP in diabetic patients $(p=0.005, r=0.38)$. The positive correlation showed that the level of the diabetic patients' hs-CRP level increases with an increase in their systolic blood pressure. Since there was a significant correlation between hs-CRP and systolic blood pressure, a linear regression model of the relationship between these two variables was assessed as $y=-1.96+0.027 \mathrm{SBP}$.

This model shows that with every unit increase in systolic blood pressure in diabetic patients, the level of
hs-CRP increases by 0.027. In this model, serum hs-CRP level can be measured based on blood pressure.

The Pearson correlation in this study indicated that there was a positive significant relationship between diabetes duration and hs-CRP in diabetic patients $(\mathrm{p}=0.03, \mathrm{r}=0.406)$. In the linear regression model, the linear correlation between the two variables was assessed as $y$ (hs-CRP) $=0.779+0.093$ Time.

This model shows that with every passing year from the development of diabetes, the level of hs-CRP increases by 0.093. In this model, hs-CRP can be measured according to the disease duration.

\section{Discussion}

The results of the present study indicated that there was a strong correlation between the level of serum hs-CRP and diastolic dysfunction, such that the higher the level of hs-CRP, the more probable the existence of diastolic dysfunction and its more advanced phases would be resulted.

There were a positive correlation and a significant relationship between diabetes duration and systolic blood pressure in diabetic patients. There was no significant relationship between hs-CRP and the variables of BMI, weight, age, HR, EF, and DBP. The results of the study also showed that there was no statistically significant relationship between the level of serum hs-CRP and gender. There was a significant relationship between diastolic dysfunction and age and disease duration. However, there was no significant relationship between diastolic dysfunction and the variables of SBP, DBP, BMI, gender, and weight.

No similar study has been carried out in Iran, and there are very few studies focusing on the relationship between biomarkers of serum hs-CRP and diastolic dysfunction.

In their prospective study, Rajaram et al. focused on the relationship between hs-CRP and diastolic dysfunction among Latin Americans during 2004-2006 in Chicago, the USA. Serum hs-CRP was directly related to diastolic dysfunction ( $\mathrm{p}=0.04, \mathrm{CI}=1.07-10.5$ (95\%), Odds Ratio=3.36). Diastolic dysfunction is prevalent among African Americans and is independently related to the increased levels of hs-CRP'.

In their study, Michowitz et al. (2006) examined the predicting role of hs-CRP in patients with heart diastolic heart failure. In that study, the level of hs-CRP was different in patients with diastolic and diastolic HF; however, after 
the symptoms alleviated, the level of hs-CRP was higher than that of healthy individuals $(\mathrm{p}<0.0001)$. In patients, this diastolic HF, the level of hs-CRP was related to NYHA classification. The results of that study indicated that the level of hs-CRP in the patients with diastolic HF is high and is related to the severity of the disease ${ }^{10}$.

In the study carried out by Amanullah in Benghazi, Libya (2010), the level of hs-CRP in diabetic and non-diabetic patients was taken into consideration. Anthropometric indices were higher in diabetic patients than non-diabetic ones. The level of hs-CRP was higher among diabetic patients. The levels of serum hs-CRP were directly related with anthropometric indices. There is a correlation between hs-CRP and sugar control with $\mathrm{HBA}_{1} \mathrm{C}$. The study indicated that there was a strong correlation between hs-CRP and diabetes ${ }^{11}$.

In the study carried out by Yorulmaz et al. in Istanbul, the predicting role of hs-CRP in the development of cardiovascular diseases among diabetic patients was taken into account. In a recent study, it was concluded that there are atleast one of the non-metabolic factors that can enhance hs-CRP, which refers to the limited use of hs-CRP as the predictor of the development risk of cardiovascular diseases among diabetic patients. There are numerous factors that can affect the level of hs-CRP; therefore, using hs-CRP has limited value as the factor to predict the risk of cardiovascular diseases among diabetic patients $^{12}$.

\section{Conclusion}

Previously conducted studies showed that diastolic dysfunction is independently associated with increased level of hs-CRP. The level of hs-CRP is high in patients suffering from $\mathrm{HF}$ diastolic and it is directly associated with the severity of the disease. According to several studies, the level of hs-CRP is higher in diabetic patients that other people and the serum level of hs-CPR is directly associated with anthropometric indices. Some other studies have introduced several factors which have the potential of affecting the level of hs-CPR; thus, applying hs-CPR as a predictor of the risk of cardiovascular disease in diabetic patients is of limited value. The present study showed that there is a considerable correspondence between the serum level of hs-CPR and diastolic dysfunction; i.e. the higher the level of hs-CPR, the higher the risk of dysfunction diastolic. There is, also, a significant relationship between the serum level of hs-CPR and duration of diabetes and systolic blood pressure. This study showed a significant relationship between diastolic dysfunction with age and duration of diabetes.

\section{References}

1. Harrison's Principles of Internal Medicine. 17th ed.US: McGraw Hill Education. 2008. Part 15, Section One, Chapter 246; Diabetes Mellitus 1463-70.

2. Shan, et al. Interleukin 18 and interleukin 18 binding protein in patients with idiopathic thrombocytopenic purpura. British J Haematology. 2009; 144(50):755-76.

3. Ridker PM, Rifai N, Clearfield M, et al. Measurement of C-reactive protein for the targeting of statin therapy in the primary prevention of acute coronary events.N Engl J Med. 2001; 344:1959-65.

4. Ridker PM, Rifai N, Rose L, Buring JE, Cook NR. Comparison of C-reactive protein and low-density lipoprotein cholesterol levels in the prediction of first cardiovascular events. N Engl J Med. 2002; 347:1557-65.

5. Danesh J, Wheeler JG, Hirschfield GM, et al. C-reactive protein and other circulating, markers of inflammation in the prediction of coronary heart disease. N Engl J Med. 2004; 350:1387-97.

6. Mahajan A, Tabassum R, Chavali S, Dwivedi OP, Bharadwaj M, Tandon N, Bharadwaj D. High-sensitivity C-reactive protein levels and type 2 diabetes in urban North Indians.J Clin Endocrinol Metab. 2009 Jun; 94(6)2123-7.

7. Nagueh SF, Appleton CP, Gillbert TC, Marino PN, et al. Recommendations for the evaluation of left ventricular diastolic function by echocardiography. Joural of American Society of Echocardiography. 2009; 22(2)103-33.

8. Otto CM. A textbook of clinical echocardiogaphy. 4th ed.US: Elsevier Health. Chapter 7; p. 157-79.

9. Rajaram V, Evans AT, Caldito GC, Kelly RF, Fogelfeld L, Black HR, Doukky R. High sensitivity C-reactive protein is associated with diastolic dysfunction in young African Americans without clinically evident cardiac disease:USA. The Open Cardiovascular Medicine Journal. 2011; 5:18895.

10. Michowitz Y, Arbel Y, Wexler D, Sheps D, Rogowski O, Shapira I, Berliner S, Keren G, George J, Roth A. Predictive value of high sensitivity CRP in patients with diastolic heart failure. Int J Cardiol. 2008 Apr 25; 125(3)347-51.

11. Amanullah S, Jarari A, Govindan M, Basha MI, Khatheeja S. Association of hs-CRP with diabetic and non-diabetic individuals. Jordan Journal of Biological Sciences. 2010; 3(1):7-12.

12. Yorulmaz E, Zunlulu MU, Alpaslan B, Oguz A. hs-CRP for cardiovascular risk in diabetes: problems in daily practice. Turkish Journal of Endocrinology and Metabolism. 2009; 2:35-8. 